\title{
Fatores de sucesso na gestão da manutenção em empresas mineradoras
}

Herbert Ricardo Garcia Viana herbertviana@hotmail.com

Universidade Federal do Rio Grande do Norte (UFRN), Natal, RN, Brasil

José Luis Duarte Ribeiro ribeiro@producao.ufrgs.br Universidade Federal do Rio Grande do Sul (UFRGS), Porto Alegre, RS, Brasil

\begin{abstract}
RESUMO
Este artigo tem como objetivo identificar, analisar e organizar os fatores determinantes para o sucesso na Gestão da Manutenção de empresas mineradoras. A identificação e organização desses fatores apoiaram-se na revisão de literatura e discussão em grupos focados. $O$ estudo em grupos focados foi desenvolvido conforme os seguintes passos: (i) planejamento; (ii) condução dos grupos focados; (iii) descrição, interpretação e análise dos dados. Como resultados, o estudo revelou 13 fatores de sucesso para a Gestão da Manutenção, entre eles estão: sistema de planejamento e controle da manutenção, engenharia da manutenção, inspeções preventiva e preditiva, sistema de gestão de riscos, sistema de custeio da manutenção, integração da manutenção e operação e melhoria continua.. A identificação dos fatores de sucesso pode contribuir positivamente no desempenho da gestão da manutenção organizando o pensamento sistêmico e, consequentemente, facilitando o entendimento das suas bases e o planejamento de ações de melhoria.
\end{abstract}

PALAVRAS-CHAVE: Gestão da Manutenção. Fatores de sucesso. Grupos focados. 


\section{INTRODUÇÃO}

O caráter estratégico da função manutenção para as empresas de transformação é defendido por vários autores. WIREMAN (1998) observa que a política de manutenção de uma empresa configura-se em um fator determinante do sucesso do planejamento da produção e, portanto, da produtividade do processo. SOURIS (1992) defende que a busca pela qualidade do processo e do produto passa pela qualidade da manutenção, sem a qual o montante investido em sistemas de gestão da qualidade pode ser inteiramente perdido.

Para KARDEC \& NASCIF (2001), a manutenção deve ser encarada como uma função estratégica na obtenção dos resultados da organização e deve estar direcionada ao suporte das atividades de gestão e de solução de problemas apresentados na produção, lançando a empresa em patamares competitivos de qualidade e produtividade.

O entendimento do peso estratégico da Função manutenção nas organizações ainda não é um ponto inteiramente aceito. MARCORIN \& LIMA (2003) afirmam que a importância da função manutenção e a opção consciente de seu modelo nem sempre são claras e levadas em consideração na análise das estratégias das organizações, e quando o são, muitas vezes acabam sendo descartadas por uma análise incorreta dos custos envolvidos.

A condição da manutenção dentro das empresas ainda mostra-se problemática. ETI et al. (2006) apontam aspectos frágeis encontrados na gestão da manutenção nas indústrias da Nigéria: falta de cultura de gestão, carência de capacitação técnica e gerencial, falta de integração com os demais processos organizacionais, baixos níveis de implementação de manutenção planejada, repetição dos problemas, trabalho errôneo da manutenção; e, mais recentemente, falha na implementação de métodos de manutenção devido a falta da consolidação dos valores e conceitos básicos inerentes a estas práticas existentes.

Segundo GURSKI \& RODRIGUES (2008), o estágio de desenvolvimento dos setores de manutenção industrial pode ser classificado segundo o grau de estruturação, sendo que grande parte das empresas não consegue impor as boas práticas mais simples, tais como o planejamento e programação de serviços ou programas de confiabilidade envolvendo manutenção preventiva ou preditiva. Essas empresas respondem de forma exaustiva às demandas sempre urgentes da produção e sofrem impactos significativos em seus orçamentos quando gerentes financeiros buscam o lucro imediato, pondo em risco o desempenho futuro.

O entendimento que os sistemas produtivos são sistemas complexos, que não se resumem apenas a Função produzir, no tocante a operação simplesmente, reforça o posicionamento estratégico da função manutenção. Logo, a determinação por parte da gestão da manutenção dos seus fatores de sucesso é um aspecto importante para possibilitar a melhoria contínua e, por conseguinte, a superação dos resultados sob sua responsabilidade.

MURTHY et al. (2002) observam que os fatores de sucesso para a gestão da manutenção estão relacionados com: os objetivos do negócio, as estratégias de manutenção, a carga de produção e o estado dos equipamentos. Conforme MARTINS \& LAUGENI (1998), os processos produtivos das empresas dependem da confiabilidade e disponibilidade de seus equipamentos e instalações, podendo 
uma planta bem mantida significar expressiva vantagem competitiva sobre a concorrência.

O presente artigo objetiva investigar quais são os fatores de sucesso mais relevantes na gestão da manutenção em empresas mineradoras e estabelecer uma estrutura para esses fatores que possa contribuir com as empresas que desejam melhorar seu desempenho junto à função manutenção.

Este artigo está organizado em seis seções. A seção 2 aborda a revisão da literatura referente a fatores de sucesso na manutenção. A Seção 3 apresenta o método de trabalho, que envolveu revisão da literatura e estudos em grupos focados. A Seção 4 apresenta a análise do trabalho desenvolvido. Por fim, a Seção 5 apresenta as principais conclusões do trabalho.

\section{REVISÃO DA LITERATURA}

A revisão da literatura aborda a importância estratégica da Função manutenção e os fatores de sucesso para a Gestão da Manutenção encontrados na literatura.

\section{IMPORTÂNCIA ESTRATÉGICA DA FUNÇÃO MANUTENÇÃO}

Segundo a NBR-5462 ABNT (1994) e BLANCHARD et al. (1995), a manutenção consiste na combinação de todas as ações necessárias para manter ou restabelecer um produto ou sistema ao estado no qual ele pode executar a função requerida, podendo incluir eventuais modificações no produto ou sistema. NAGAO (1999) observa que o impacto de uma manutenção inadequada e ineficiente pode definir a rentabilidade do negócio e a sobrevivência do empreendimento. Segundo SOUSA et al. (2011), atualmente a manutenção está presente no dia-a-dia das organizações, sendo de suma importância para garantir o desempenho funcional do equipamento, atribuindo dessa forma, confiabilidade ao processo produtivo.

Para KARDEC \& NASCIF (2001), a missão da manutenção é garantir a disponibilidade da função dos equipamentos e instalações de modo a atender a um processo de produção ou de serviço com confiabilidade, segurança, preservação do meio ambiente e custos adequados. Também segundo esses autores, a manutenção deve ser encarada como uma função estratégica na obtenção dos resultados da organização e deve estar direcionada ao suporte das atividades de gestão e de solução de problemas apresentados na produção, lançando a empresa em patamares competitivos de qualidade e produtividade.

O caráter estratégico da Função manutenção para as empresas de transformação é defendido por vários autores. WIREMAN (1998) observa que a política de manutenção de uma empresa configura-se em um fator determinante do sucesso do planejamento da produção e, portanto, da produtividade do processo. SOURIS (1992) defende que a busca pela qualidade do processo e do produto passa pela qualidade da manutenção, sem a qual o montante investido em sistemas de gestão da qualidade pode ser inteiramente perdido.

O entendimento do peso estratégico da Função manutenção nas organizações ainda não é um ponto inteiramente aceito. MARCORIN \& LIMA 
(2003) afirmam que a importância da Função manutenção e a opção consciente de seu modelo nem sempre são claras e levadas em consideração na análise das estratégias das organizações, e quando o são, acabam sendo descartadas por uma análise incorreta dos custos envolvidos.

Observa-se na literatura um reconhecimento sobre a importância estratégica da manutenção no desempenho das empresas (NAGAO, 1999; KARDEC \& NASCIF, 2001; WIREMAN, 1998 e SOURIS, 1992). Ao mesmo tempo, constata-se a dificuldade das empresas incorporarem essa importância (MARCORIN \& LIMA, 2003), o que também é observado nos resultados da pesquisa nacional ABRAMAN do setor em 2011, em particular aqueles referentes a alocação de recursos em manutenção corretiva e indisponibilidade de ativos por manutenção.

\section{FATORES DE SUCESSO NA GESTÃO DA MANUTENÇÃO}

A pesquisa nacional da ABRAMAN realizada em 2011 mostra uma série de dados que retratam o panorama nacional da gestão da manutenção. Valorizamse na pesquisa dados como a formação educacional dos profissionais e resultados da manutenção expostos através de indicadores como Disponibilidade Operacional e Indisponibilidade. Além destes, a pesquisa aborda com maior ênfase os valores relativos aos Custos de manutenção.

Observam-se no direcionamento da pesquisa alguns dos fatores de sucesso para gestão da manutenção considerando a qualificação de uma boa ou má gestão de acordo com os resultados obtidos nos mesmos, são eles: estrutura organizacional da manutenção; custos do setor; qualificação do pessoal; política de terceirização e uso das ferramentas da qualidade para análise de falha.

Outras fontes indicam fatores distintos ou complementares. Segundo TSANG (2002), são quatro os fatores de sucesso para o gerenciamento da manutenção: (i) Modelo de serviço, interno com pessoal próprio ou externo com contratação de trabalhos terceirizados em fornecedores especializados; (ii) Organização da manutenção em seu organograma; (iii) Metodologia da manutenção com a definição das políticas a serem adotadas e (iv) Infraestrutura de suporte à função manutenção. Tais fatores são considerados os direcionadores da gestão, envolvendo entradas como: mão-de-obra, materiais, almoxarifado, ferramental, sistema de informações e custeio. Essas entradas são capazes de gerar as saídas de tal processo, as quais podem ser avaliadas através de indicadores como disponibilidade dos equipamentos, segurança no trabalho e lucros do negócio.

WIREMAN (1989) afirma que o gerenciamento da manutenção deveria contemplar dez fatores: Manutenção preventiva, Inventário e compras, Sistema de ordens de trabalho, Sistema de gerenciamento da manutenção computadorizado, Treinamento técnico e interpessoal, Envolvimento operacional, Manutenção pró-ativa, RCM, TPM, e Melhoria contínua.

MARQUEZ \& GUPTA (2006) observam que a gestão determina os objetivos da manutenção ou prioridades, bem como a definição e implementação de métodos de melhoria, incluindo aspectos econômicos da organização.

WIREMAN (1989) e INGALLS (2001) apresentam uma visão sobre o que vem a ser uma Manutenção Classe Mundial. Segundo o último, a mesma repousa nas melhores práticas de manutenção observadas em doze fatores: Liderança e 
Política, Estrutura Organizacional, Controle de Inventários, Sistemas Computadorizados de Administração, Manutenção Preventiva, Manutenção Preditiva, Planejamento e Programação, Fluxo de Trabalho, Controle Financeiro, Envolvimento das Pessoas, Recursos Humanos e Treinamento, e Melhoramento Contínuo. Nota-se na exposição do autor o estabelecimento de fatores determinantes para o sucesso da gestão da manutenção. Segundo o mesmo, os doze fatores trabalhados em seu artigo são determinantes para o sucesso na gestão da manutenção.

WIREMAN (1998) organiza os fatores que influenciam a gestão da manutenção em uma pirâmide, lançando a noção da necessidade de se ter uma base sólida para suportar pontos mais avançados. $\mathrm{O}$ autor hierarquiza os fatores de manutenção estipulando aqueles que formam a base e os de fases mais avançadas que serão suportados pelos mais básicos, conforme ilustra a Figura 1.

Figura 1 - Pirâmide da Manutenção

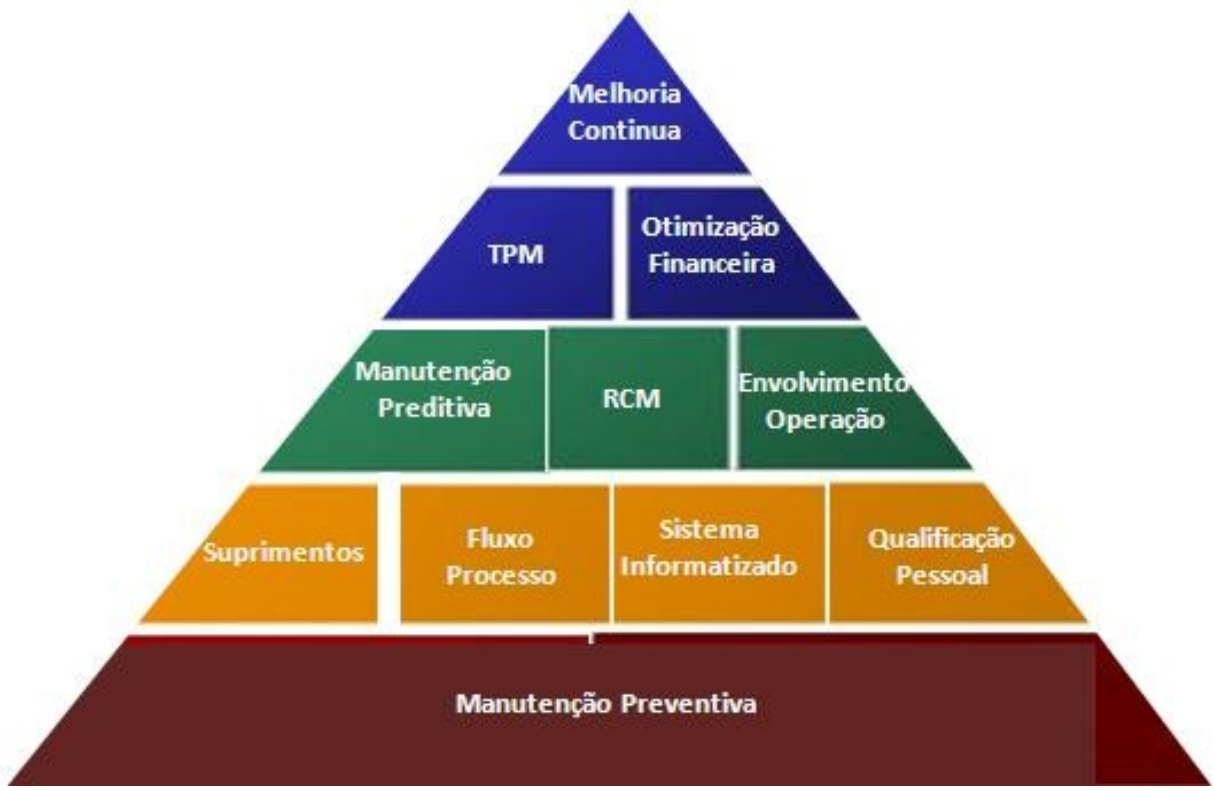

Fonte: Wireman (1998)

HATAKEYAMA et al. (2006) indicam que os fatores de sucesso para a implantação de uma manutenção de qualidade total são: Produtividade, Custos, Suprimentos, Qualidade de serviços para o atendimento do retrabalho zero, Segurança no trabalho e Moral da equipe através da participação de todos. Além dos pontos citados, os autores reconhecem a importância de um sistema de auditoria dos processos de manutenção que garanta um correto feedback para a equipe através da visualização de possíveis pontos de melhoria que possam reforçar o desempenho das gerências de manutenção.

NYMAN \& LEVITT (2001) organizam os fatores de sucesso da gestão da manutenção na forma de um arco, semelhante à pirâmide de Wireman. A Figura 2 ilustra a lógica defendida por esses autores. Percebe-se que a pedra fundamental que sustenta o arco de Nyman e Levitt são os fatores "manutenção preventiva" e "manutenção preditiva". Da mesma forma, a base da pirâmide de Wireman é o fator "manutenção preventiva". Constate-se, assim, a preocupação dos autores em alicerçar a gestão da manutenção em seu processo básico de 
realização de serviços antes da ocorrência da falha. Esta abordagem é relevante na medida em que sugere que nenhum avanço na função manutenção terá sustentabilidade, caso não se tenha um fundamento sólido nas atividades básicas da manutenção, como aquelas que garantem a adequada aplicação de uma manutenção preventiva.

Figura 2 - Arco da gestão da manutenção

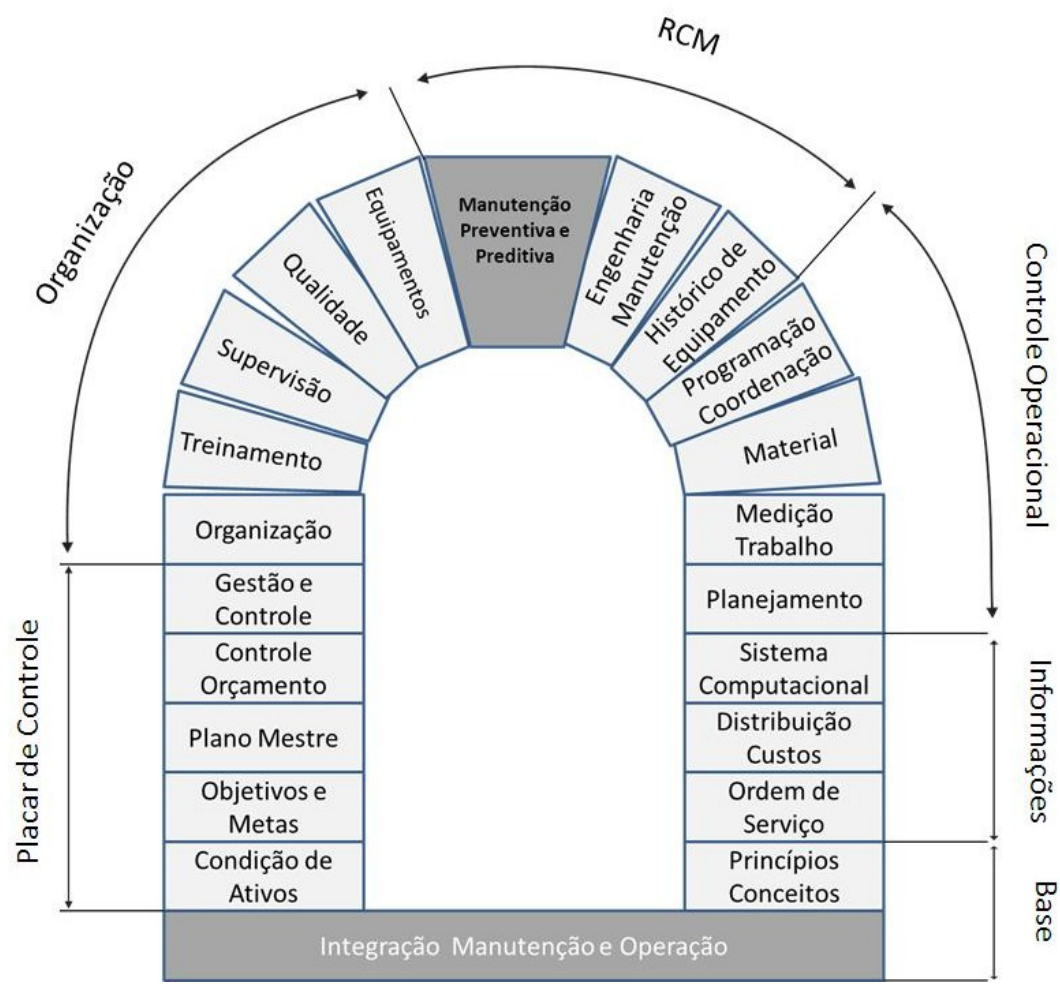

Fonte: Nyman e Levitt (2001)

CALLIGARO (2003) avalia que os fatores de sucesso na gestão da manutenção repousam naquilo que o mesmo chama de quatro fundamentos, sendo eles: (i) Planejamento e programação da manutenção, (ii) Otimização da demanda de serviços de manutenção, (iii) Engenharia de manutenção e (iv) Qualidade na execução dos serviços de manutenção.

A revisão da literatura sobre os fatores de sucesso na gestão da manutenção indica várias listas onde existe variação de nomenclatura e diferenças de abrangência (alguns autores apresentam visões mais amplas, outros mais detalhada). De qualquer forma, através de um esforço de síntese, é possível organizar os fatores listados na literatura conforme apresentado no Quadro 1.

Quadro 1 - Fatores de sucesso na gestão da manutenção observados na literatura

\begin{tabular}{|ccc|}
\hline Seq & Fatores determinantes & Referências \\
\hline \multirow{2}{*}{1} & Sistemas computadorizados de & WIREMAN, 1989; WIREMAN, 1998; \\
& administração da manutenção & INGALLS, 2001; NYMAN; LEVITT, 2001 \\
& & INGALLS, 2001; NYMAN; LEVITT, 2001; \\
2 & Sistema de Planejamento e Controle & CALLIGARO, 2003; BRANCO FILHO, 2008; \\
& da manutenção & MACEDO et al., 2008; GURSKI et al., 2008; \\
& & HAARMAN; DELAHAY, 2004.
\end{tabular}


3 Sistema de custeio da manutenção

4

Estrutura organizacional definida para o setor da manutenção

5

Sistema de Segurança no trabalho e saúde ocupacional

6

7

Sistema de monitoramento das

8

condições - Inspeções Preventiva e Preditiva

9

Capacitação das pessoas da manutenção

10

Suprimentos de materiais

11

Integração da manutenção e operação

12
ABRAMAN, 2011; INGALLS, 2001;

HATAKEYAMA et al., 2006; WIREMAN,

1998; NYMAN; LEVITT, 2001; HAARMAN; DELAHAY, 2004

ABRAMAN, 2011; TSANG, 2002; INGALLS, 2001; NYMAN; LEVITT, 2001

HATAKEYAMA et al. 2006; CALLIGARO, 2003; HAARMAN; DELAHAY, 2004; MUNIZ, 2010; KARDEC; NASCIF, 2001; GURSKI et al., 2008; LINHARES; GARCIA, 2004 NYMAN; LEVITT, 2001; CALLIGARO, 2003; HAARMAN; DELAHAY, 2004; MÁRQUEZ; GUPTA, 2006; VIANA, 2002.

ABRAMAN, 2011; WIREMAN, 1989; WIREMAN, 1998; INGALLS, 2001; HAARMAN; DELAHAY, 2004

WIREMAN, 1989; WIREMAN, 1998; INGALLS, 2001; NYMAN; LEVITT, 2001

ABRAMAN, 2011; WIREMAN, 1989; WIREMAN, 1998; INGALLS, 2001; NYMAN; LEVITT, 2001; XAVIER, 2005; GONZALES et al., 2012; SALERMO, 2005; KARDEC; NASCIF, 2001

HATAKEYAMA et al., 2006; WIREMAN, 1998; NYMAN; LEVITT, 2001; CALLIGARO, 2003; MALLMANN, 2000)

WIREMAN, 1989; WIREMAN, 1998; INGALLS, 2001; NYMAN; LEVITT, 2001; XAVIER, 2005

INGALLS, 2001; HATAKEYAMA et al., 2006; XAVIER, 2005

Fonte: Autoria própria (2017)

Cabe ressaltar que a nomenclatura utilizada na Figura 3 corresponde a uma compilação dos vários estudos pesquisados. Percebem-se pequenas diferenças na terminologia dos fatores de sucesso na gestão da manutenção reportados na literatura.

\section{MÉTODO}

O trabalho desenvolvido seguiu uma abordagem qualitativa, apoiando-se principalmente em conceitos apresentados na literatura e opinião de especialistas, reunida através de estudos em grupos focados. Quanto aos objetivos, a pesquisa desenvolvida pode ser classificada como descritiva, uma vez que descreve em detalhe os fatores de sucesso na gestão da manutenção. Quanto aos procedimentos, a pesquisa caracteriza-se como bibliográfica e pesquisa-ação. Bibliográfica porque a estrutura proposta foi fortemente baseada em conceitos apresentados na literatura; pesquisa-ação porque o desenvolvimento foi realizado em estreita associação com a resolução de um 
problema coletivo, onde o pesquisador e profissionais da empresa que serviu de objeto de estudo estiveram envolvidos de modo cooperativo.

A identificação dos fatores determinantes para o sucesso da gestão da manutenção apoiou-se na revisão de literatura e discussão em grupos focados. A primeira etapa envolveu a revisão bibliográfica sobre o tema, através da consulta de artigos e livros disponíveis no ambiente acadêmico. A revisão produziu uma lista de fatores de sucesso, consolidada através da compilação dos elementos que se apresentaram em comum em duas ou mais das referencias consultadas.

Em paralelo foram realizados grupos focados. O estudo em grupos focados foi desenvolvido conforme os seguintes passos: (i) planejamento; (ii) condução dos grupos focados; (iii) descrição, interpretação e análise dos dados. O tamanho dos grupos foi definido de forma a evitar grupos muito pequenos, pois são facilmente dominados por um ou dois membros, ou grupos muito grandes que podem causar tédio em alguns participantes ou gerar conversas paralelas, como observa CHURCHIL \& NIELSEN, s.d.: SIMON (1999). Assim os grupos tiveram a participação de seis a oito pessoas.

Os grupos foram divididos conforme o nível hierárquico de atuação dos seus membros, buscando desta forma evitar possível predominância de membros baseada na sua posição de ascendência sobre os demais. A aplicação de grupos focados envolveu sessões com três grupos distintos: gerentes de área, supervisores e engenheiros/analistas. O roteiro de perguntas era simples, baseado em dois questionamentos: (i) $\mathrm{Na}$ sua visão quais são os fatores determinantes para o sucesso da gestão da manutenção? (ii) Quais são os ganhos para uma empresa onde os fatores de sucesso estejam presentes?

A segunda pergunta buscava incentivar os membros do grupo a vincularem os fatores indicados com resultados observados na prática industrial. Esse procedimento estimulava os participantes a revisar suas indicações, buscando perceber alguma incoerência lógica, do tipo: se é um fator de sucesso, por que não gera ganho?

De posse das informações dos grupos focados e da revisão da literatura, os dados reunidos foram organizados, interpretados e analisados, gerando assim uma primeira lista de fatores de sucesso na gestão da manutenção.

Em seguida, realizou-se o retorno aos mesmos grupos focados para discussão da lista organizada dos fatores de sucesso, buscando compreender a influência desses fatores no ambiente específico da empresa em estudo. Esse segundo momento contribuiu para validar a lista de fatores de sucesso através de uma discussão cujo cenário era um ambiente prático e conhecido dos participantes. Este fato permitiu capturar as percepções dos membros dos grupos focados e proceder à análise dos resultados reunidos.

O estudo em grupos focados foi desenvolvido conforme os seguintes passos: (i) planejamento; (ii) condução dos grupos focados; (iii) organização e análise dos dados, conforme orientam BEYEA \& NICOLL (2000) apud RIBEIRO \& NEWMANN (2003).

No planejamento foram definidos: (i) os objetivos do grupo, no caso investigar o entendimento de profissionais da manutenção industrial sobre os fatores de sucesso da função; (ii) o roteiro de perguntas; (iii) o tamanho dos grupos e sua distribuição por níveis hierárquicos; (iv) o tempo de duração das 
sessões; (v) o local adequado para as discussões e (vi) a forma de registro das informações.

Na condução dos grupos preocupou-se na criação de um ambiente propício para captura de interpretações livres sobre quais seriam os fatores de sucesso da gestão da manutenção, tendo o cuidado no direcionamento dos debates, para evitar perdas de rumo durante as argumentações. Na medida do possível, o moderador evitou apresentar opiniões que poderiam induzir o pensamento condicionado a um conceito preestabelecido pelo mesmo.

Os dados fundamentais produzidos foram então transcritos das discussões do grupo, acrescidos das anotações e reflexões do moderador. $O$ trabalho de análise dos dados iniciou-se por uma tabulação dos fatores de sucesso elencados por cada grupo focado, relacionando-os com as argumentações apresentadas que sustentaram sua escolha como fator de sucesso durante os debates. Em seguida, houve a compatibilização das tabelas geradas por cada grupo, gerando uma lista única, a qual recebeu contribuição do entendimento do pesquisador sobre o tema.

O roteiro de perguntas era simples, baseado em três questões centrais, conforme dispostos no Quadro 2.

Quadro 2 - Roteiro de perguntas na aplicação dos grupos focados

\begin{tabular}{|c|c|}
\hline Sequência & Descrição da pergunta \\
\hline $1 \underline{a}$ & $\begin{array}{l}\text { Na sua visão quais são os fatores estratégicos determinantes para } \\
\text { o sucesso da gestão da manutenção? }\end{array}$ \\
\hline $2 \underline{a}$ & $\begin{array}{l}\text { Na sua visão quais são os fatores de rotina determinantes para o } \\
\text { sucesso da gestão da manutenção? }\end{array}$ \\
\hline $3 \underline{a}$ & $\begin{array}{l}\text { Quais são os ganhos para uma empresa onde os fatores de sucesso } \\
\text { estejam presentes? }\end{array}$ \\
\hline
\end{tabular}

Fonte: Autoria própria (2017)

A primeira pergunta foi formulada para ser direta e capaz de impulsionar os participantes do grupo focado em direção a respostas que traduzam o universo da manutenção nos quais estão inseridos, bem como na expressão da sua experiência adquirida em diversos setores de manutenção. A utilização de uma questão aberta repousa na intenção de induzir uma maior participação dos entrevistados.

A segunda pergunta objetivou funcionar como um elemento regulador, capaz de provocar nos entrevistados a diferenciação dos fatores estratégicos daqueles fundamentos da rotina da manutenção, bem como identificar algum fundamento de rotina tão presente e necessário para uma estrutura de manutenção que o faria um fator de sucesso para a função.

A terceira pergunta buscava incentivar os membros do grupo a vincularem os fatores indicados com resultados observados na prática industrial. Esse procedimento estimulava os participantes a revisar suas indicações, buscando perceber alguma incoerência lógica, do tipo: se é um fator de sucesso, por que não gera ganho? 
Além disso, a disposição das perguntas favoreceu uma ordem crescente de profundidade, conforme orienta RIBEIRO \& NEWMAN (2003). Dessa forma, o envolvimento dos entrevistados se intensificava ao logo das discussões. O estímulo ao trabalho coletivo fez com que as percepções e conceitos fossem se moldando dentro de um senso comum criado pelo próprio grupo. Temas abertos proporcionam em um primeiro momento uma diversidade de entendimentos, mas, sob um espaço de pensamento comum, permitem explorar a fundamentação dos entendimentos diversos para se chegar em consensos, que no início dos trabalhos do grupo poderiam parecer improváveis.

O tamanho dos grupos foi definido de forma a evitar grupos muito pequenos, pois são facilmente dominados por um ou dois membros, ou grupos muito grandes, que podem causar tédio em alguns participantes ou gerar conversas paralelas, como observa CHURCHIL \& NIELSEN, s.d.: SIMON (1999). Assim, os grupos tiveram a participação de seis a oito pessoas.

Os grupos foram divididos conforme o nível hierárquico de atuação dos seus membros, buscando desta forma evitar uma possível predominância de membros baseada na sua posição de ascendência sobre os demais. Assim, a aplicação de grupos focados envolveu sessões com três grupos distintos: gerentes de área, supervisores e engenheiros, todos profissionais atuando na empresa de mineração.

\section{RESULTADOS}

A organização dos dados foi realizada basicamente na compilação dos fatores de sucessos elencados por cada grupo em tabelas. O Quadro 3 apresenta os fatores determinados no grupo dos gerentes; o Quadro 4 apresenta os resultados do grupo de supervisores e o Quadro 5 resume os pensamentos do grupo de engenheiros. As listas apresentadas nessas figuras já foram processadas de forma a eliminar problemas de terminologia.

Quadro 3 - Fatores de sucesso observados no grupo de Gerentes da empresa mineradora

\begin{tabular}{|cc|}
\hline Seq & Fatores de sucesso para Gestão da manutenção \\
\hline 1 & Sistemas computadorizados de administração da manutenção \\
2 & Sistema de Planejamento e Controle da manutenção \\
3 & Sistema de custeio da manutenção \\
4 & Estrutura organizacional definida para o setor da manutenção \\
5 & Sistema de Segurança no trabalho e saúde ocupacional \\
6 & Engenharia da manutenção \\
7 & Círculos de controle da qualidade \\
8 & Manutenção Preditiva \\
9 & Capacitação das pessoas da manutenção \\
10 & Suprimentos de materiais \\
11 & Integração da manutenção e operação
\end{tabular}


12

13

14

15
Sistema de gestão de riscos (confiabilidade)

Sistema de Gestão Ambiental

Política da Automação industrial

Sistema de investimentos na manutenção

Fonte: Autoria própria (2017)

Quadro 4 - Fatores de sucesso observados no grupo de Supervisores da empresa mineradora

\begin{tabular}{|cc|}
\hline Seq & Fatores de sucesso para Gestão da manutenção \\
\hline 1 & Sistemas computadorizados de administração da manutenção \\
2 & Sistema de Planejamento e Controle da manutenção \\
3 & Sistema de custeio da manutenção \\
4 & Sistema de Segurança no trabalho e saúde ocupacional \\
5 & Capacitação das pessoas da manutenção \\
6 & Suprimentos de materiais \\
7 & Sistema de Gestão de pessoas \\
8 & Engajamento e Motivação \\
\hline
\end{tabular}

Fonte: Autoria própria (2017)

Quadro 5 - Fatores de sucesso observados no grupo de Engenheiros da empresa mineradora

\begin{tabular}{|cc|}
\hline Seq & Fatores de sucesso para Gestão da manutenção \\
\hline 1 & Sistemas computadorizados de administração da manutenção \\
2 & Sistema de Planejamento e Controle da manutenção \\
3 & Sistema de custeio da manutenção \\
4 & Sistema de Segurança no trabalho e saúde ocupacional \\
5 & Engenharia da manutenção \\
6 & Melhorias e Modificações de Equipamentos \\
7 & Manutenção Preditiva \\
8 & Capacitação das pessoas da manutenção \\
9 & Gestão de contratos (Terceirização) \\
10 & Sistema de gestão de riscos (confiabilidade) \\
11 & Sistema de Gestão Ambiental \\
12 & Política da Automação industrial \\
\hline
\end{tabular}


Fonte: Autoria própria (2017)

Os resultados da literatura e dos grupos individuais foram objeto de análise em uma segunda fase do estudo de grupos focado. Nessa segunda fase, um grupo formado por três gestores, três engenheiros e três supervisores analisou o conjunto de resultados anteriores e gerou uma lista final de fatores de sucesso na Gestão da manutenção em empresas mineradoras.

A lista final reúne treze fatores de sucesso, dispostos no Quadro 6. Os mesmos permeiam o universo da manutenção em suas mais variadas nuances, englobando de forma ampla aquilo que tem peso estratégico para a Função manutenção no ambiente das empresas mineradoras. Considerando a opinião dos especialistas consultados e apoiado na literatura, acredita-se que a atenção a esses fatores permite à manutenção obter resultados adequados em seu desempenho operacional e sustentáveis ao longo do tempo. A atenção a esses fatores ainda contribui para a construção de um ambiente motivador para o exercício profissional dos colaboradores do setor de manutenção.

Quadro 6 - Fatores de sucesso na Gestão da Manutenção em empresas mineradoras

\begin{tabular}{|cc|}
\hline Seq & Fatores de sucesso para Gestão da manutenção \\
\hline 1 & Sistemas computadorizados de administração da manutenção \\
2 & Sistema de Planejamento e Controle da manutenção \\
3 & Sistema de custeio da manutenção \\
4 & Sistema de Segurança no trabalho e saúde ocupacional \\
5 & Engenharia da manutenção \\
6 & Sistema de gestão de pessoas: capacitação e motivação \\
7 & Melhoria continua \\
8 & Estrutura organizacional definida para o setor da manutenção \\
9 & Preditiva \\
10 & Sistema de monitoramento das condições - Inspeções Preventiva e \\
11 & Sistema de Gestão Ambiental \\
12 & \\
13 &
\end{tabular}

Fonte: Autoria própria (2017)

Observando a lista dos fatores de sucesso apresentados no Quadro 7, notase que os mesmos podem ser classificados em dois blocos distintos, seguindo uma lógica de sequenciamento de implantação e planejamento de atividades na 
gestão de uma área de manutenção. 0 primeiro bloco refere-se à estrutura básica necessária para a operacionalização da gestão da manutenção. Estabelecida essa estrutura básica, o segundo bloco trata da operacionalização propriamente dita da gestão da manutenção. O sequenciamento entre os fatores oferece uma lógica daquilo que deve ser a base da gestão da manutenção, oferecendo o conceito de avanço, para as empresas que desejam implantar a base e progredir em direção a resultados de classe mundial na manutenção.

A Estrutura Organizacional é um dos fatores de sucesso na gestão da manutenção, observado tanto na literatura como nos grupos focados. Há consenso na literatura em não haver um modelo fechado para a função manutenção que permita distribuir em um organograma os recursos humanos de um departamento de manutenção, visto que cada empresa possui sua especificidade.

Para PERES \& LIMA (2008), mobilizar e dominar a sustentabilidade da estratégia na manutenção depende das pessoas, que são os principais ativos a serem considerados. Assim, o sistema de gestão de pessoas constitui o segundo fator de sucesso do bloco básico. XAVIER (2005) observa que, para que a empresa atinja a excelência, é necessária a melhoria em todas as áreas, e isso só será obtido pelo engajamento e colaboração de toda a equipe.

Quadro 7 - Fatores de sucesso sequenciados em blocos

\begin{tabular}{ll}
\hline Estrutura básica \\
\hline 1 & Estrutura organizacional definida para o setor da manutenção \\
2 & Sistema de gestão de pessoas: capacitação e motivação \\
3 & Sistema de Segurança no trabalho e saúde ocupacional \\
4 & Sistema de Gestão Ambiental \\
5 & Suprimentos de materiais \\
\hline Operação, avaliação e melhoria \\
\hline 6 & Sistemas computadorizados de administração da manutenção \\
7 & Sistema de Planejamento e Controle da manutenção \\
8 & Engenharia da manutenção \\
& Sistema de monitoramento das condições - Inspeções Preventiva e \\
9 & Preditiva \\
10 & Sistema de gestão de riscos (confiabilidade) \\
11 & Sistema de custeio da manutenção \\
12 & Integração da manutenção e operação \\
13 & Melhoria continua \\
\hline
\end{tabular}

Fonte: Autoria própria (2016)

$\mathrm{Na}$ literatura a segurança é identificada como uma das dimensões mais utilizadas nos indicadores de desempenho industrial. Assim, esse é o terceiro fator de sucesso do bloco básico. Os grupos focados também perceberam este fator de sucesso, demonstrando assim um compromisso cultural dos profissionais da manutenção com a segurança dos mantidos e mantenedores.

Outro fator de sucesso destacado nesta pesquisa junto ao bloco básico foi aquele que abriga a preocupação dos profissionais da manutenção com o 
equilíbrio com o mundo exterior, no caso, o Sistema de Gestão Ambiental. Esse sistema vem crescendo em importância nas diversas empresas produtoras brasileiras, visto o peso estratégico que o tema incorporou nos últimos anos não só por questões comerciais, mas também por questões morais inerentes aos seres humanos.

Observa-se que os fornecedores exercem grande impacto em todos os fatores importantes para a estratégia de operações. Tais impactos no universo da manutenção ocorrem no quesito qualidade dos serviços que não geram retrabalhos, pois uma peça fornecida sem a devida qualidade nas suas especificações pode ser motivo de intervenções corretivas e custos significativos associados a paradas de produção ou acidentes com consequências materiais, ambientais ou humanas. Dessa forma, o Suprimento de Materiais aparece como o último fator do bloco básico.

A seguir são descritos os fatores do bloco referente à operação, avaliação e melhoria, esses diretamente associados às atividades de manutenção.

Dentre os fatores elencados neste segundo bloco, os "Sistemas Computadorizados de Administração" estão presentes como elementos de sucesso não só na Função manutenção, mas em várias vertentes da administração das empresas, visto a importância do tratamento dos dados para assegurar agilidade da tomada de decisão. Assim, como em outras áreas, um sistema computadorizado adequado para fornecer suporte na administração da manutenção é considerado essencial.

O Planejamento e Controle da Manutenção constituem o segundo fator de sucesso do bloco operacional. Essas atividades tornam-se primordiais a partir da exigência do aumento da competitividade atrelada à condição de sobrevivência para as empresas. Esse cenário exige a busca do máximo retorno financeiro sobre os ativos industriais, através do aumento da disponibilidade para a produção, mantendo custos de manutenção adequados. Sem um planejamento eficiente, a ocorrência de erros e perdas no trabalho da manutenção certamente será maior.

A Engenharia de Manutenção é o terceiro fator de sucesso do bloco operacional. Esta deve ter constante preocupação com a melhoria da manutenabilidade. As pessoas da engenharia de manutenção são as responsáveis por realizar as análises de criticidade e desenvolver a melhoria dos procedimentos da manutenção. MÁRQUEZ \& GUPTA (2006) observam que os bons resultados da Função manutenção são indicações claras da existência de uma série de boas práticas, dentre elas uma engenharia de manutenção atualizada e competente.

O quarto fator de sucesso do bloco operacional, Monitoramento de Condições, pode ser encarado como o conjunto de tarefas da manutenção preventiva e preditiva cujo objetivo é a previsão de falhas através do monitoramento e controle dos parâmetros operacionais. O sistema de monitoramento é percebido como um dos alicerces da gestão da manutenção (WIREMAN, 1998; NYMAN \& LEVITT, 2001).

A confiabilidade, retratada no quinto fator de sucesso do bloco operacional, encontra ampla sustentação na literatura que aborda a gestão da manutenção. Em alguns casos, sua presença é detectada na própria definição do que é Manutenção, conforme KARDEC \& NASCIF (2001), os quais definem manutenção 
como a função que garante a disponibilidade dos equipamentos e instalações de modo a atender a um processo de produção e à preservação do meio ambiente, com CONFIABILIDADE, segurança e custos adequados.

O Sistema de Custeio é o sexto fator de sucesso do bloco operacional. Esse sistema tem grande importância visto que os custos relacionados à manutenção são uma das prioridades das empresas. A redução de custos de manutenção pode repercutir em aumento dos lucros operacionais. Assim, o desenvolvimento de sistemas que monitorem os custos da manutenção e auxiliem na tomada de decisão é outro aspecto essencial.

A Integração da Manutenção e da Operação é o sétimo fator de sucesso. Isso acontece porque manter a boa condição de ativos não é uma missão exclusiva da manutenção, mas deve envolver aqueles que estão no cotidiano operacional. Daí surge a necessidade de integrar manutenção e operação, estabelecendo relações que favoreçam o alcance do resultado desejado.

Por fim, o último fator do bloco operacional contempla a Melhoria Contínua. No âmbito da manutenção, isso implica estabelecer um cenário (cultura organizacional) onde todos estão engajados sistematicamente na elevação da confiabilidade dos ativos e na sua melhor utilização para o atendimento dos objetivos das organizações produtivas.

\section{CONCLUSÃO}

O presente artigo investigou os fatores de sucesso na gestão da manutenção em empresas mineradoras, pesquisando-os de forma a definir um conjunto organizado de elementos que podem ser considerados os mais relevantes no universo da função manutenção.

O trabalho desenvolvido seguiu uma abordagem qualitativa, apoiando-se principalmente em conceitos apresentados na literatura e na opinião de especialistas, reunida através de estudos em grupos focados.

Através da revisão da literatura e estudo em grupos focados, foi construída uma lista de 13 fatores de sucesso. Esses fatores foram organizados em dois blocos: (i) estrutura básica e (ii) operação, avaliação e melhoria. Os fatores de sucesso identificados junto à estrutura básica foram cinco: estrutura organizacional definida para o setor da manutenção; sistema de gestão de pessoas: capacitação e motivação; sistema de segurança no trabalho e saúde ocupacional; sistema de gestão ambiental; e suprimentos de materiais. Os fatores de sucesso identificados junto ao bloco de operação, avaliação e melhoria foram oito: sistemas computadorizados de administração da manutenção; sistema de planejamento e controle da manutenção; engenharia da manutenção; sistema de monitoramento das condições - inspeções preventiva e preditiva; sistema de gestão de riscos (confiabilidade); sistema de custeio da manutenção; integração da manutenção e operação; melhoria continua.

A lista de fatores de sucesso pode contribuir de maneira positiva no desempenho da gestão da manutenção em mineradoras, uma vez que organiza o pensamento sistêmico sobre a função manutenção, favorecendo o entendimento das suas bases e o planejamento de ações para atender satisfatoriamente cada um dos fatores de sucesso elencados. 
Apesar dos fatores de sucesso identificados neste artigo serem fruto da opinião de especialistas de uma empresa mineradora, observa-se que a estrutura proposta deve ser válida em outros setores em que a presença da função manutenção é determinante para os resultados produtivos, observando-se a necessidade de pequenas adaptações para aplicações em outros setores. 


\title{
Success Factors in Maintenance Management in Mining Companies
}

\begin{abstract}
This paper aims to identify, analize and organize the key factors for success in Maintenance Management of mining companies. The identification and organization of these factors were based on a literature review and focus group studies. The focus groups were conducted according to the following steps: i) planning, ii) focus group conduction, iii) description, interpretation and analysis. As a result, the study reveal 13 success factors for Maintenance Management, among them are: maintenance planning and control system, maintenance engineering, preventive and predictive inspections, risk management system, maintenance cost system, maintenance-operation integration, and continuous improvement system. The identification of the success factors can help to improve the maintenance management by organizing its systemic thinking and, consequently, improving the action planning process.
\end{abstract}

KEYWORDS: Maintenance Management. Success Factors. Focus Group. 
ASSOCIAÇÃO BRASILEIRA DE MANUTENÇÃO (ABRAMAN). Documento Nacional. Curitiba: ABRAMAN, 2011.

ASSOCIAÇÃO BRASILEIRA DE NORMAS TÉCNICAS (ABNT). NBR 5462: confiabilidade e mantenabilidade. Rio de Janeiro: ABRAMAN, 1994.

ASSOCIAÇÃO BRASILEIRA DE NORMAS TÉCNICAS (ABNT). NBR ISO/IEC 9126 Tecnologia de informação - Avaliação de produto de software, Características de qualidade e diretrizes para o seu uso. Rio de Janeiro: 1996.

CALLIGARO, C.. Proposta de fundamentos habilitadores para a gestão da manutenção em indústrias de processamento contínuo baseada nos princípios da manutenção classe mundial. Dissertação do Programa de Pós-Graduação em Engenharia de Produção, UFRGS, 2003.

CHURCHILL JUNIOR, G. A.; NIELSEN JUNIOR, A. C. Marketing Research, Methodological Foundations. 6 ed. The Dryden Press, s.d. Cap. 4, p.153-161: Research Design, 1999.

ETI, M. C.; OGAJI, S. O. T.; PROBERT, S. D.. Development and implementation of preventive-maintenance practices in Nigerian industries. Applied Energy, v. 83, p. 1163-1179, 2006. crossref

GONZALEZ, I. V. D. P.; LIMA, C. R. C.; BATISTA, J. A.; PELISSARI, A. S.. Processo de aprendizagem no contexto da gestão de manutenção visando à confiabilidade. Revista Gestão Contemporânea, v.2, n.1, 2012.

GURSKI C. A.; RODRIGUES M.. Planejando estrategicamente a manutenção. XXVIII XXVIII Encontro Nacional de Engenharia de Produção, Rio de Janeiro: ENEGEP, 2008.

HAARMAN, M.; DELAHAY, G.. Value driven maintenance (VDM): New faith in maintenance. Holanda: Mainnovation, 2004.

HATAKEYAMA, K.; RODRIGUES, M.. Analysis of the fall of TPM in companies. Journal of Materials Processing Technology, 2006.

KARDEC, A.; NASCIF, J., Manutenção Função Estratégica. Rio de Janeiro: Editora Qualitymark, 2001. 
LINHARES, A.; GARCIA, P. C.. Contratos de terceirização de manutenção industrial modalidade risco: análise das expectativas do setor industrial e dos provedores de serviços. Revista de Administração Mackenzie, ano 05, no 02, p. 79-98, 2004.

MARCORIN, W. R.; LIMA, C. R. C.. Análise dos custos de manutenção e de nãomanutenção de equipamentos produtivos. Revista de Ciência \& Tecnologia, v. 11, no 22, p. 35-42, 2003.

MÁRQUEZ, A. C.; GUPTA, J. N. D.. Contemporary maintenance management: process, framework and supporting pillars. International Journal of Management Science, v. 34, p. 313-326, 2006.

MARTINS, P. G.; LAUGENI, F. P.. Administração da Produção. São Paulo: Saraiva, 1998.

MUNIZ, R. P. D.. Requisitos de mantenabilidade na execução dos serviços de manutenção. Dissertação do Programa de Pós-Graduação em Engenharia de Produção, UFRGS, 2010.

MURTHY D. N. P.; ATRENS, A.; ECCLESTON J. A.. Strategic maintenance management. Journal of Quality in Maintenance Engineering. n. 2, v. 8, p. 287305. 2002. crossref

NAGAO, S. K.. Manutenção Industrial - análise, diagnóstico e propostas de melhoria de performance em indústrias de processo. XIV Congresso Brasileiro de Manutenção, Foz do Iguaçu: ABRAMAN, 1999.

NYMAN, D.; LEVITT, J.. Maintenance planning, scheduling and coordination. New York: Industrial Press Inc., 2001.

PERES, C. R. C.; LIMA, G. B. A.. Análise do Capital Organizacional da Manutenção sob a Perspectiva do Aprendizado e Crescimento do Balanced Scorecard. Congresso Nacional de Excelência em Gestão, Niterói: UFF, 2008.

RIBEIRO, J. ; NEWMANN, C.. Planejamento e condução de grupos focados. In: RIBEIRO, J. (Org.) Grupos focados: teoria e aplicações. Porto Alegre: FEENGUFRGS-PPGEP, 2003.

SALERNO, M. S.; AULICINO, M. C.. Engenharia, manutenção e operação em processos contínuos: elementos para o projeto de fronteiras organizacionais móveis e interpenetrantes, Revista Gestão Produção, v. 15, n. 2, p. 337-349, 2008. crossref 
SIMON, J. S.. How to conduct focus group. Nonprofit Word. Madison, v.17, n.5, p. 40-43, 1999.

SOURIS, J. P., Manutenção Industrial - custo ou benefício. Tradução de Elizabete Batista. Lisboa: Lidel, 1992.

SOUSA, E. P. M. et al.. Gestão da manutenção no setor de tecnologia de informação de uma Universidade federal. VI Simpósio de Engenharia de Produção da Região Nordeste, Campina Grande, 2011.

TSANG, A. H. C.. Strategic dimensions of maintenance management. Journal of Quality in Maintenance Engineering, v. 8, n.1, p. 7-39, 2002. crossref

WIREMAN, T.. World Class Maintenance Management. Proceedings of the Automatic Factory Conference, Detroit, United States, 1989.

WIREMAN, T.. Developing Performance Indicators for Managing Maintenance. Nova York: Industrial Press, 1998.

XAVIER, J. N.. Manutenção Classe mundial. Congresso Brasileiro de Manutenção. Salvador: ABRAMAN, 2005. Acessado pelo site http://www.tecem.com.br/downloads/manutencao.pdf em 01/05/2012.

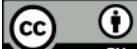

